Annuaire suisse de politique de développement

13 | 1994

Annuaire Suisse - Tiers Monde 1994

\title{
V. Politique économique extérieure
}

\section{(2) OpenEdition}

1 Journals

Édition électronique

URL : http://journals.openedition.org/aspd/1032

DOI : 10.4000/aspd.1032

ISSN : 1663-9669

Éditeur

Institut de hautes études internationales et du développement

\section{Édition imprimée}

Date de publication : 1 janvier 1994

Pagination : 112-134

ISSN : 1660-5934

\section{Référence électronique}

« V. Politique économique extérieure », Annuaire suisse de politique de développement [En ligne], 13|

1994, mis en ligne le 06 décembre 2012, consulté le 08 septembre 2020. URL : http://

journals.openedition.org/aspd/1032 ; DOI : https://doi.org/10.4000/aspd.1032

(c) The Graduate Institute I Geneva 


\section{Politique économique extérieure}

\section{Garantie contre les risques à l'exportation}

En 1992, pour la première fois depuis 1978, la garantie contre les risques à l'exportation a clos son exercice annuel par un excédent. Ce résultat s'explique par l'activation de crédits de consolidation auprès de pays en développement. Le compte des liquidités s'est encore soldé par un déficit qui a à nouveau dû être couvert par un prêt de la Confédération. Les avances fédérales consenties à la GRE se montaient ainsi à la fin de 1992 à 2,47 milliards de francs. Les 96\% des nouvelles garanties concernent des pays en développement et des pays de l'Est.

La garantie contre les risques à l'exportation (GRE) est un instrument à l'usage des exportateurs suisses, qui leur permet d'assurer auprès de la Confédération certains risques inhérents à l'exportation. Cette assurance instituée en 1934 était à l'origine destinée à sauvegarder des places de travail. Par la suite, elle est devenue un instrument de la Confédération pour favoriser le commerce extérieur. La base légale de l'assurance actuelle a été créée en 1958 (loi fédérale sur la GRE du 26.9.1958). La part des exportations garanties par la GRE représente 2,66\% (1992) de l'ensemble des exportations suisses. Les pays en céveloppement absorbent $16 \%$ des exportations suisses et la part des pays de l'Est est de $3 \%$. Environ $80 \%$ des exportations suisses sont destinées aux pays de l'OCDE.

La GRE permet à l'exportateur de s'assurer contre des risques que ni lui, ni l'acheteur ne peuvent influencer, ainsi que contre l'insolvabilité d'acheteurs ou de garants publics. Voici les risques couverts:

- Risques politiques: une guerre ou des troubles empêchent l'exécution de l'obligation contractuelle.

- Risques de transfert: Le client est dans l'impossibilité de payer la dette en raison d'une mesure gouvernementale sur le marché des changes. II peut avoir déposé 
la contre-valeur en monnaie locale, mais la banque centrale n'est pas à même de fournir les devises nécessaires.

- Risque de consolidation des dettes: Risque que des échéances déterminées d'un pays se trouvant confronté à de graves difficultés financières soient rééchelonnées, c'est-à-dire repoussées de plusieurs années, sur la base d'une convention internationale.

- Risque de ducroire: Risque d'insolvabilité ou de refus de paiement lorsque les clients sont des acheteurs de droit public (Etats, communes, institutions de droit public, sociétés d'utilité publique telles que centrales électriques, entreprises d'incinération des déchets, etc.) et lorsqu'une institution publiquea fourniunegarantie.

- Risque de fabrication: Risque qu'une marchandise ne puisse être livrée suite à des événements politiques ou des mesures d'un Etat étranger. Cela implique la couverture spécifique du risque avant livraison.

La couverture des risques monétaires ou de change a été suspendue en 1985. Ne sont en outre pas couverts le risque d'insolvabilité d'un débiteur privé, les dommages qui font suite à une réclamation légitime pour des défauts, les risques de transports. Lagarantie des risques à l'exportation n'est accordée qu'à des entreprises qui sont établies en Suisse et qui sont inscrites au Registre du commerce.

Le Bureau de la GRE reçoit les demandes et les soumet, après examen préalable, à la Commission. La Commission est composée de 8 membres: 3 représentants de la Confédération, 3 représentants de l'économie privée, ainsi que, depuis que le nombre des sièges a été augmenté le 1 er mars 1992, représentante des employés et 1 représentant de la coopération publique au développement (DDA). Les oeuvres privées d'entraide qui souhaitaientégalement pouvoir siéger au sein de la Commission ne sont pas représentées.

Lorsque la somme garantie ne dépasse pas 1 million de francs, l'instance compétente pour décider de l'octroi de la GRE est l'Office fédéral des affaires économiques extérieures (Département fédéral de l'économie publique, DFEP); lorsqu'elle se situe entre 1 et 2 millions de francs, c'est le DFEP qui décide et au-delà de 2 millions le DFEP décide avec l'accord du Département fédéral des finances. Les demandes qui revêtent une importance particulière sont soumises au Conseil fédéral.

\section{Réassurance par des programmes spéciaux de la Confédération}

Lagrande majorité des contrats garantis est passée avec des pays en développement et des pays de l'Est. En 1992, 96\% des nouvelles garanties ont été accordées pour des pays de ce groupe et seuls $4 \%$ pour des pays de l'OCDE. La GRE tient à jour une liste de pays à haut risque pour lesquels aucune garantie n'est accordée. En 1992, les garanties ne pouvaient ainsi pas être accordées dans 45 pays ou n'étaient octroyées que dans le domaine du court terme pour 44 autres pays. La liste des pays exclus de la GRE comprend par exemple la Corée du Nord, Cuba, la Syrie, ainsi que des pays d'Afrique noire avec lesquels la Suisse n'entretient guère de relations économiques. La sécurité économique est le critère de base pour l'octroi de la garantie contre les risques à l'exportation. Nombre de pays de l'Est ne sont donc pas 
encore admis dans la GRE, bien que la Suisse collabore avec eux dans le domaine de la coopération au développement. Dans certains cas particuliers, la GRE peut toutefois aussi être demandée pour ces pays-là. La Confédération assume alors directement le risque, tandis que la GRE se charge des modalités administratives. Les moyens sont prélevés sur divers crédits-cadres de la coopération destinés au développement et à l'aide à l'Est.

Un programme de ce type a été ouvert dans le cadre de l'aide à l'Est et il comprend les pays suivants: les Etats baltes, la Bulgarie, la Croatie, la Pologne et la Slovénie. Un programme pour les Etats de la CEI est en préparation. Un autre programme couvre les crédits mixtes accordés aux pays en développement. Pour pouvoir continuer à accorder des crédits mixtes à des pays en principe fermés à la GRE, la Confédération peut puiser dans les crédits-cadres destinés à financer la politique économique et commerciale, ainsi que dans le crédit de 400 millions de francs adopté en 1991, à l'occasion du 700e anniversaire de la Suisse, pour financer des mesures de désendettement. La part fédérale des crédits mixtes doit se monter à $35 \%$ au moins. Dans les nouveaux crédits mixtes, elle constitue un don.

\section{Autofinancement}

La GRE devrait devenir économiquement autonome, mais elle s'est sensiblement écartée de cet objectif dans les années 80 , car elle a dû verser d'importantes indemnités en raison de la crise de l'endettement. Les créances de consolidation accumulées ont été réévaluées (1992: 50\%) et représentent la majeure partie des actifs du bilan de la GRE. De 2,5 milliards en 1991, elles sont passées à 3,2 milliards en 1992. L'objectif de l'autonomie économique est inscrit dans la loi, afin que les prix de la GRE suivent les prix du marché, c'est-à-dire que les primes varient en fonction des risques. Par ailleurs, les risques présentant une forte probabilité de pertes sont exclus de la garantie. La politique prudente de la GRE en matière de risque est en contradiction - surtout en temps de récession - avec la stimulation de l'emploi. Compte tenu du ralentissement économique dans les pays industrialisés, la concurrence s'est en particulier renforcée sur les marchés des pays nouvellement industrialisés et l'octroi de la GRE joue un rôle important pour l'économie suisse d'exportation.

\section{Prestations de la GRE en 1992}

En 1992, la GRE a octroyé de nouvelles garanties pour un montant total de 1,9 milliard de francs (valeur facturée:2,3 milliards) contre 1,7 milliard l'année précédente. Le total des engagement s'est légèrement accru pour atteindre 8,2 milliards. Les tableaux $\mathrm{n}^{0} 11$ et $\mathrm{n}^{0} 12$ indiquent la répartition régionale des nouvelles garanties. Cette répartition reflète la force économique d'une région et l'intérêt dont fait preuve la Suisse à commercer avec les pays concernés. La majeure partie des garanties $(67 \%)$ a été octroyée à des pays d'Asie, $12 \%$ à des pays d'Amérique centrale et du Sud, $15 \%$ à des pays d'Afrique, $5 \%$ à des pays du Sud de l'Europe et $2 \%$ à des pays de l'Est. 
Le rapport annuel de la GRE consacre un chapitre particulier au groupe des 67 pays en développement les plus défavorisés. La GRE ayant été octroyée à un grand projet de construction hydro-électrique en Indonésie, la part de ce groupe a atteint $46 \%$ des garanties de la GRE (32,6\% en $1991,21,7 \%$ en 1990). Les années précédentes pourtant, les garanties de la GRE se concentraient également sur quelques-uns de ces pays qui présentent un intérêt économique pour la Suisse. Depuis sa révision en 1981, la loi prévoit que les contrats conclus avec des pays en développement et couverts par la GRE doivent aussi respecter les principes du développement.

Tableau $\mathrm{N}^{\circ} 11$

Répartition géographique des nouvelles garanties

\begin{tabular}{|c|c|c|c|c|}
\hline \multirow{2}{*}{ Groupes de pays } & \multicolumn{2}{|c|}{1992} & \multicolumn{2}{|c|}{1991} \\
\hline & en mio. fr. & part en $\%$ & en mio. fr. & part en $\%$ \\
\hline Europe & 90,3 & 4,8 & 262,1 & 15,6 \\
\hline Europe orientale & 33,3 & 1,7 & 75,1 & 4,5 \\
\hline Amérique du Nord & - & - & - & - \\
\hline Amérique du Sud / Centrale & 223,8 & 11,8 & 153,8 & 9,2 \\
\hline Afrique & 277,3 & 14,6 & 254,1 & 15,1 \\
\hline Asie & $1 ' 273,2$ & 67,1 & 931,7 & 55,6 \\
\hline TOTAL & $1 ' 897,9$ & 100,0 & $1 ' 676,8$ & 100,0 \\
\hline dont: & & & & \\
\hline Pays membres de l'OCDE & 74,9 & 3,9 & 253,0 & 15,1 \\
\hline $\begin{array}{l}67 \text { pays les plus défavorisés } \\
\text { dont: }\end{array}$ & 873,9 & 46,0 & 546,3 & 32,6 \\
\hline Amérique du Sud / Centrale & - & - & 2,5 & 0,2 \\
\hline Afrique & 92,7 & 4,8 & 99,4 & 5,9 \\
\hline Asie & 781,2 & 41,2 & 444,4 & 26,5 \\
\hline
\end{tabular}

Source: Rapport annuel 1992 de la GRE, Berne/Zurich, juin 1993

La part des pays de l'Est dans les nouvelles garanties de la GRE a encore diminué par rapport à l'année précédente. Cette baisse s'explique par la diminution des exportations vers ces pays où règne l'insécurité politique et économique. La nouvelle hausse de la part de l'Amérique centrale et du Sud doit principalement être imputée à la forte demande de garanties pour le Mexique, ainsi qu'à la réouverture de la GRE à l'Argentine pour le moyen et le long terme. Les garanties pour les pays africains concernent essentiellement des pays du Nord et du Sud (Afrique du Sud) de ce continent. Les pays en développement et les pays asiatiques nouvellement industrialisés représentent deux tiers des nouvelles garanties. Une garantie a 
Tableau $\mathrm{N}^{\circ} 12$

\begin{tabular}{|c|c|c|c|c|}
\hline \multicolumn{5}{|c|}{ Répartition géographique du total des engagements } \\
\hline \multirow[b]{2}{*}{ Groupes de pays } & \multicolumn{2}{|c|}{1992} & \multicolumn{2}{|c|}{1991} \\
\hline & $\begin{array}{c}\text { en mio. } \\
\text { fr. }\end{array}$ & $\begin{array}{l}\text { part } \\
\text { en } \%\end{array}$ & $\begin{array}{c}\text { en mio. } \\
\text { fr. }\end{array}$ & $\begin{array}{l}\text { part } \\
\text { en } \%\end{array}$ \\
\hline Europe & $1 ' 236,6$ & 15,1 & $1 ' 335,8$ & 16,6 \\
\hline Pays de l'Europe de l'Est & 750,1 & 9,1 & 972,6 & 12,0 \\
\hline Amérique du Nord & 41,2 & 0,5 & 43,9 & 0,5 \\
\hline Amérique du Sud / Centrale & $1^{\prime} 628,9$ & 20,0 & $1 ' 726,5$ & 21,4 \\
\hline Afrique & $1^{\prime} 526,5$ & 18,6 & $1 ' 498,1$ & 18,4 \\
\hline Asie & $3^{\prime} 002,3$ & 36,7 & $2^{\prime} 496,8$ & 31,1 \\
\hline TOTAL & $8^{\prime} 185,6$ & 100,0 & $8^{\prime} 073,7$ & 100,0 \\
\hline dont: & & & & \\
\hline Pays membres de l'OCDE & $1^{\prime} 121,1$ & 13,7 & $1 ' 195,0$ & 14,8 \\
\hline $\begin{array}{l}67 \text { pays les plus défavorisés } \\
\text { dont: }\end{array}$ & $2 ' 302,0$ & 28,1 & $1 ' 751,5$ & 21,7 \\
\hline Amérique du Sud / & & & & \\
\hline Centrale & 2,5 & 0,0 & 22,7 & 0,4 \\
\hline Afrique & 622,1 & 7,6 & 623,7 & 7,7 \\
\hline Asie & $1 ' 677,4$ & 20,5 & $1^{\prime} 105,1$ & 13,6 \\
\hline
\end{tabular}

Source: Rapport annuel de la GRE, Berne/Zurich, juin 1993

notamment été accordée à un grand projet en Indonésie. L'Indonésie fait partie des pays en développement les plus défavorisés, de sorte que la part de ce groupe de pays s'est sensiblement accrue en 1992.

Pour ce qui est de la répartition par branches, l'industrie des machines a bénéficié de $78 \%$ des garanties et la chimie de $20 \%$, ce qui correspond à la répartition de l'année précédente. Les grands contrats pour lesquels une garantie a été demandée sont surtout le fait de grandes entreprises. Asea Brown Boveri (ABB), qui est engagé dans des projets de construction de grandes centrales hydro-électriques, est le plus gros client de la GRE. Selon des articles parus dans la presse, ABB a obtenu des garanties pour 4 milliards de francs, soit la moitié environ de l'engagement total de la GRE (1).

Résultat de l'exercice 1992

Pourlapremière fois depuis 1978 (àl'exception de 1986 où le systèmed'amortissement a été modifié), l'exercice 1992 de la GRE révèle un excédent qui se monte à 140 
millions de francs. Ce résultat positif s'explique par l'activation de dommages de transfert des années précédentes, qui ont fait l'objet d'accords de consolidation avec les pays débiteurs. Le taux de réévaluation s'est monté à $50 \%$. La balance du flux monétaire se solde en 1992 de nouveau par un déficit de liquidités; celui-ci atteint 204 millions de francs ( 335 millions l'année précédente) et il a été couvert par un nouveau prêt de la Confédération. Le total des avances accordées par la Confédération à la GRE atteint ainsi 2,47 milliards de francs.

\section{Versement d'indemnités}

En 1992, la GRE a versé 118 millions de francs à titre d'indemnités. Le Brésil vient en tête des pays pour lesquels des indemnités ont été payées, avec 52 millions de francs. Suivent la Yougoslavie (19 millions de francs), l'Irak (13), l'Argentine (13), I'URSS (12), la Bulgarie (4), le Nigéria, la Pologne et le Honduras (chacun 1 million de francs environ). Selon le Bureau de la GRE, le montant des indemnités versées devrait diminuer ces prochaines années, car les paiements sur d'anciennes garanties qui datent des années 80 , c'est-à-dire de la crise de l'endettement, arrivent à terme. D'un autre côté, de nouvelles "sources de dommages" apparaissent dans les pays de l'Est.

\section{Désendettement}

Selon la décision prise en 1990 par le Parlement pour assainir la GRE (2), les mesures de désendettement en faveur de pays en développement doiventégalement alléger le bilan de la GRE. Lorsque, dans le cadre d'actions de désendettement, la GRE cède à la Confédération ses avoirs de consolidation envers un pays en développement, la Confédération accorde à la GRE une avance d'un montant équivalent. En 1992, dans l'optique de la réalisation de telles actions, la Confédération a racheté aux exportateurs et aux banques les franchises des créances destinées au désendettement. Les prix payés lors de cette opération suivaient ceux du niveau du marché secondaire correspondant. Les franchises, dont la valeur nominale s'élevait à 304 millions de francs, ont été rachetées pour 59 millions (soit 19\%). Les parts correspondantes de la GRE se montent à 780 millions de francs. La plupart des exportateurs ont participé à ces opérations. La valeur nominale totale des créances ayant bénéficié d'actions de désendettement s'élève à 1 '084 millions de francs. Sur la base de telles actions de rachat, la GRE peut compter sur une remise des avances de la Confédération pour un montant d'environ 780 millions de francs, dès que des accords de rééchelonnement auront été conclus avec les différents pays.

\section{Livraison d'appareils Pilatus à l'Afrique du Sud: garantie controversée}

La Communauté de travail des grandes oeuvres d'entraide suisses à invité le Conseil fédéral à refuser la demande de garantie présentée pour couvrir la livraison d'appareils Pilatus à l'Afrique du Sud (60 PC-7 pour l'aviation sud-africaine). Selon elle, la Suisse se devait de respecter l'embargo sur les armes décrété par l'ONU à l'encontre de ce pays. En juin, le Conseil fédéral a approuvé cette livraison (d'un 
montant total de 194 millions de francs). Une part de 45 millions de ce montant est assurée par la GRE (3). Le Conseil fédéral a motivé sa décision en déclarant que la Suisse n'étant pas membre de l'ONU, elle n'était pas tenue de respecter l'embargo. Dans la statistique des exportations, les appareils Pilatus sont classés parmi le matériel civil et leur livraison à l'étranger peut bénéficier de la GRE. La GRE ne peut toutefois pas être accordée pour des livraisons de matériel de guerre (4).

\section{Notes}

1. Tages-Anzeiger, 17.8.1993 (ABB construit une centrale en Libye).

2. La Confédération a été obligée de consentir une avance totale de 900 millions de francs pour éponger les pertes engendrées par la couverture de la variation du taux de change. Pour alléger les comptes de la GRE, le Parlement à autorisé la Confédération à renoncer pour 10 ans aux intérêts sur cette avance de 900 millions de francs. Pour plus de détails sur l'assainissement de la GRE, voir le chapitre GRE dans l'Annuaire Suisse-Tiers Monde 1991.

3. Tages-Anzeiger du 2.6.1993 (L'Afrique du Sud peut acheter des PC-7) et NZZ du 4.5.1993 (Les oeuvres d'entraide s'opposent à l'octroi de la garantie pour la vente d'appareils Pilatus).

4. Au sujet de l'utilisation militaire des appareils Pilatus lire le chapitre "Exportations d'armes" du présent Annuaire.

\section{Sources}

Rapport annuel de la GRE 1992, Zurich/Berne, juin 1993

Bureau de la GRE, Zurich

Rapport sur la politique économique extérieure, 1992/1+2

Office fédéral des affaire économiques extérieures

NZZ, 29.6.1993 (Comptes de la GRE)

Tages-Anzeiger, 4.5.1993 (Les oeuvres d'entraide disent non à la GRE pour les ateliers Pilatus)

Tages-Anzeiger, 2.6.1993 (L'Afrique du Sud peut acheter des PC-7)

Comité pour le désarmement et l'arrêt des exportations d'armes (éd.), Friedenspolitik $n^{0}$ 68/juin 1993 (Le scandale des PC-7 perdure)

\section{Préférences douanières}

Dans leur effortd'intégration au sein du commerce moridial, les pays en développement libéralisent et ouvrent leurs marchés. La libéralisation du commerce international se heurte cependant toujours au prélèvement de droits de douane et, de plus en plus, à des obstacles non tarifaires, tels que les contingents. Ces dernières années pourtant, de grands progrès ont été réalisés en matière de suppression des droits de douane, de sorte que ce sont aujourd'hui surtout les obstacles non tarifaires qui freinent le commerce international. Le système généralisé de préférences (SGP), mis en place par la CNUCED en 1968, a beaucoup contribué à réduire les droits de douane; un Comité spécial des préférences veille au respect du SGP et contrôle chaque année son application. Dans le cadre du GATT, la suppression des droits de douane représente aussi un objectif important des négociations de l'Uruguay Round. 
Une réduction des droits de douane sert à favoriser les exportations de produits manufacturés des pays en développement, pour leur permettre de mettre sur pied une industrie de transformation et de "se libérer ainsi de leur dépendance à l'égard des produits de base, dont le faible dynamisme et les fluctuations de prix importantes provoquent des déficits chroniques de balance courante." Voilà les termes utilisés par le Conseil fédéral dans son message de 1991 (1). Dans le cadre du SGP, les pays industrialisés accordent des préférences douanières unilatérales et autonomes à des pays en développement. Autrement dit, les droits de douane ne font pas l'objet de négociations, les pays industrialisés accordent des préférences sans exiger la réciprocité. On dénombre actuellement 16 schémas préférentiels. Les plus importants sont ceux accordés par la CEE, le Japon et les Etats-Unis. La Suisse a commencé à appliquer un schéma préférentiel pour les pays en développement en 1972. Au total, 160 pays en développement et de l'Est bénéficient d'un allégement des droits de douane grâce aux divers schémas préférentiels.

Il est pourtant possible d'améliorer encore les effets que les schémas préférentiels en vigueur exerce sur le commerce. Environ $50 \%$ des importations provenant de pays en développement dans les pays industrialisés mériteraient un traitement de faveur. Dans la pratique, le système n'a pas encore réalisé tout son potentiel étant donné que, selon la CNUCED, les importations préférentielles sont restées à un niveau de $25 \%$ environ des importations passibles de droits. En Suisse, le taux des importations préférentielles s'est élevé en 1992 à 43\% (voir tableau no 13). Ce taux relativement faible s'explique par le fait que les pays en développement connaissent mal l'utilisation des 16 différents schémas préférentiels dans le cadre du SGP et par l'importance du coût administratif, la complexité de l'obligation de notifier (règles d'origine) et par la quantité de restrictions des différentsschémas. Deplus, la "diplomatie économique", très coûteuse, dépasse souvent les moyens des pays en développement, surtout des plus défavorisés. La Suisse soutient la coopération technique fournie par le Secrétariat de la CNUCED aux pays en développement pour leur permettre d'améliorer l'utilisationdesschémaspréférentiels. Cette coopération consiste par exemple à financer des cours de formation régionaux ou à fournir un soutien technique.

Laclause delagradation permetd'exclurecertains paysendéveloppement ou certains produits du SGP lorsqu'ils sont suffisamment concurrentiels face aux pays industrialisés. Dansuntelcas, les lois du marchédoivent pouvoir s'appliquer. Les pays endéveloppement demandentàprésent,quelagradation-sielledoitêtreappliquée-soitrégiepardescritères clairs résultant d'un consensus multilatéral. Ils souhaitent ainsi éviter une application arbitraire de cette clause. Avant d'exclure un pays ou un produit du système préférentiel, ilseraitnotammentessentieldeprouverqu'iljouiteffectivementd'unecapacitéconcurrentielle durable. Pour cela, on devra consulter le pays concerné et respecter un certain délai. Jusqu'à présent, la Suisse n'a jamais appliqué la clause de la gradation. II est vrai qu'en 1972, lorsqu'elle a mis en oeuvre son schéma préférentiel, elle n'a pas complètement exempté de droits de douane certains produits pour lesquels les pays en développement étaient particulièrement concurrentiels, mais ne leur a octroyé qu'une réduction des droits de $50 \%$. II s'agit notamment des textiles, des vêtements, des chaussures à semelle en cuir, des parapluies, des piles électriques et de l'aluminium brut. Ce principe est partie intégrante duschémapréférentieldelaSuisseetn'estpas considérécommeuneclause de gradation. 
A l'occasion de l'examen annuel de l'application du SGP en mai 1993, quelques pays en développement ont demandé au Secrétariat de la CNUCED de mener une étude sur l'impact de l'élargissement du SGP aux pays de l'Est.

Le représentant de la Chine a vivement regretté une pratique qui tend à se généraliser et qui consiste à soumettre l'octroi d'un traitement préférentiel à des critères qui n'ont aucun rapport avec le commerce, tels que le respect des droits de I'homme, la politique de l'environnement et les droits des travailleurs. II estime que ces mesures violent le principe de la non-discrimination du SGP.

Le développement futur du SGP dépend pour beaucoup de l'issue des négociations du cycle d'Uruguay dans le cadre du GATT. On s'attend à des allégements dans le commerce de produits agricoles (tarification et suppression du protectionnisme) et à un élargissement de la liste des produits finis et semi-finis pouvant bénéficier de traitements préférentiels (2). La politique du SGP subira une révision fondamentale pour être adaptée à l'évolution commerciale d'ici à 1995.

\section{Le schéma préférentiel suisse}

Depuis le milieu des années 80 , la part des importations en provenance de pays en développement dans l'ensemble des importations suisses stagne entre 7 et $8 \%$,

Tableau $\mathrm{N}^{\circ} 13$

Importations suisses et préférences tarifaires 1990-1992

\begin{tabular}{|l|c|c|c|}
\hline Année & 1990 & 1991 & 1992 \\
\hline $\begin{array}{l}\text { 1. Volume total des importations de la Suisse, } \\
\text { en millions de francs }\end{array}$ & $96^{\prime} 611$ & $95^{\prime} 032$ & $92^{\prime} 330$ \\
$\begin{array}{l}\text { 2. Importations en provenance des pays en voie } \\
\text { de développement, en millions de francs }\end{array}$ & $77^{\prime} 610$ & $7^{\prime} 411$ & $66^{\prime} 616$ \\
$\begin{array}{l}\text { 3. Part des importations en provenance des PVD } \\
\text { en \% (point 2 par rapport au point 1) }\end{array}$ & 7,88 & 7,80 & 7,70 \\
$\begin{array}{l}\text { 4. Importations pouvant bénéficier du traitement } \\
\text { préférentiel, mio. de fr. }\end{array}$ & $5 ' 308$ & $5 ' 148$ & $4{ }^{\prime} 666$ \\
$\begin{array}{l}\text { 5. Importations ayant effectivement bénéficié } \\
\text { des préférences, mio. de fr. }\end{array}$ & $2 ' 009$ & $2 ' 066$ & $2^{\prime} 009$ \\
$\begin{array}{l}\text { 6. Degré d'utilisation des avantages accordés } \\
\text { en \% (point 5 par rapport au point 4) }\end{array}$ & 37,85 & 40,13 & 43,05 \\
\hline \multicolumn{2}{|l|}{ Source: BAWI } & \\
\hline
\end{tabular}


tandis que les exportations suisses vers ces pays atteignent $16 \%$ environ du total des exportations. Au début des années 80 , la part des pays en développement dans le commerce extérieur était légèrement plus élevée.

LaSuisse aadopté un schéma de préférences pour les importations en provenance des pays en développement en 1972. II prévoit la libre importation pour la plupart des biens industriels, à l'exception des produits énumérés ci-dessus qui sont soumis à un traitement spécial. La situation est toute autre dans le secteur agricole, car la Suisse protège l'agriculture nationale par des obstacles commerciaux non tarifaires (tels que l'imposition de quotas d'importation) et prélève des droits de douane sur les importations de produits agricoles. Depuis 1982, elle accorde toutefois un traitement différencié aux pays en développement les plus défavorisés: une centaine de produits agricoles et industriels peuvent être importés librement en Suisse.

En 1992, les importations totales de la Suisse se sont montées à quelque 92 milliards de francs et les importations en provenance des pays en développement à 6,6 milliards, ce qui correspondà $7,17 \%$. Le traitement préférentiel n'a effectivement été utilisé que pour l'importation de marchandises d'une valeur de 2 milliards de francs, ce qui correspond à un taux d'utilisation de $43 \%$ des importations pouvant bénéficier du schéma préférentiel (voir tableau $\mathrm{n}^{0} 13$ ).

Le schéma de préférences suisse est régulièrement remis à jour. En 1992, les pays ci-après ont ainsi été admis sur la liste des pays les plus défavorisés au bénéfice d'un traitement de faveur: Libéria, Cambodge, Madagascar, îles Salomon, Zambie, Zaïre. Cette liste compte à présent 47 pays.

L'Albanie a été admise parmi les pays bénéficiant de préférences douanières en 1992. Depuis cette même année, la Bulgarie et la Roumanie profitent aussi de tous les avantages du schéma de préférences suisse. En 1993, la Suisse a conclu avec la Roumanie un accord de libre-échange, ce qui a annulé l'application des préférences douanières. La Turquie est dans le même cas, car un accord de libre-échange est aussi entré en vigueur entre ce pays et I'AELE dont la Suisse fait partie. La Suisse a reconnu la Croatie, la Slovénie et la Bosnie-Herzégovine comme Etats souverains et les a inscrits sur la liste des pays au bénéfice des préférences douanières. Elle s'est toutefois ralliée à l'embargo de l'ONUà l'encontre de la Serbie et du Monténégro. Les marchandises en provenance de "Macédoine" jouissent de préférences douanières.

Pour déterminer les pays bénéficiaires de son système de préférences, la Suisse applique le principe de l'auto-élection (elle admet tout pays en développement qui se présente comme tel). L'Albanie constitue une exception, car ce pays ne s'est pas déclaré lui même en développement. Au milieu de 1993, l'admission de certains pays de l'Est demeurait en question. On envisage notamment d'admettre dans le schéma préférentiel des pays d'Asie centrale qui remplissent les conditions de la coopération au développement (et que la Suisse représente dans un groupe de vote au sein du Fonds monétaire international).

Le Conseil fédéral songe aussi à étendre les avantages octroyés aux pays en développement, surtout aux moins avancés d'entre eux, afin de tenir compte "de leur niveau de développement modeste et de leur degré de diversification économique toujours très faible ainsi que de leur intégration encore peu poussée dans le système commercial mondial" (cf. note 1). 


\section{Notes}

1. Message du Conseil fédéral concernant la prolongation de l'arrêté fédéral du 9 octobre 1981 sur l'octroi de préférences tarifaires en faveur des pays en développement, 20.2.1991 (91.017).

2. CNUCED, Communiqué de presse, 10.5.1993 (Examen de l'application du Système généralisé de préférences).

\section{Sources}

CNUCED, Communiqué de presse (TAD/INF/2327 du 10 mai 1993), Examen du SGP CNUCED, Rapport général sur l'application du système généralisé de préférences, 4 mars 1993

Rapport concernant les mesures tarifaires prises durant le 2ème trimestre 1992, Berne, 20.1.1993 (93.007)

Message du Conseil fédéral concernant la prolongation de l'arrêté fédéral du 9 octobre 1981 sur l'octroi de préférences tarifaires en faveur des pays en développement, 20.2.1991 (91.017)

Office fédéral des affaires économiques extérieures

\section{Entretiens économiques}

Les relationséconomiques de la Suisse avec les pays en développementcomprennent pour l'essentiel des relations commerciales et des investissements directs. Parmi les bénéficiaires des investissements on trouve principalement le Brésil, le Mexique et l'Argentine; tandis que la majeure partie des échanges commerciaux font intervenir des pays en développement du continent asiatique. En 1991, les importations suisses en provenance de pays en développement se sont montées à 7,3 milliards de francs et à 6,5 milliards en 1992. Quant aux exportations, elles ont représenté 14,2 milliards de francs en 1991 et 15,4 en 1992. La majeure partie des échanges commerciaux concernent le groupe des "pays exportateurs de produits manufacturés à croissance rapide". II s'agit des sept pays suivants: Mexique, Brésil, Corée du Sud, Taiwan, Singapour, Hong-Kong et, jusqu'en 1991, la Yougoslavie. Par ailleurs, l'approvisionnementénergétique-importations d'Arabie saoudite, d'Iran, des Emirats arabes et de Libye (jusqu'à l'embargo décrété en 1991) - occupe une part importante dans les échanges commerciaux.

Pour promouvoir les relationséconomiques etl'ouverture de nouveaux débouchés ou pour trouver de nouveaux clients, la Suisse entretient une diplomatie économique qui consiste à envoyer des délégations mixtes, comprenant des représentants de l'administration et de l'économie privée, dans les pays concernés ou à inviter en Suisse des délégations de pays en développement. Les relations économiques peuvent également prendre la forme de participation à des foires commerciales internationales. La Suisse contribue à financer des chambres de commerce avec ses principaux partenaires économiques; celles-ci ont pour tâche de stimuler les échanges bilatéraux. 
Voici les contacts qui ont marqué la période considérée (juillet 1992 à septembre 1993):

Asie

En juillet 1992, une délégation mixte emmenée par le Conseiller fédéral Jean-Pascal Delamuraz s'est rendue en Chine. Les deux pays ont conclu un accord sur la protection de la propriété intellectuelle. Un accord de protection des investissements est en vigueur depuis 1987 et une convention de double imposition depuis 1991 (cf. Annuaire 1993).

En juillet 1993, la Suisse a établi des relations économiques avec un nouveau partenaire en Asie: le Viêt-nam. Une délégation économique a séjourné pendant une semaine dans le pays. Elle comptait vingt membres, dont des représentants de l'administration fédérale et de l'économie privée, et avait à sa tête Nicolas Imboden, délégué du Conseil fédéral aux accords commerciaux. La délégation avait pour tâche de définir le cadre juridique de futures relations économiques. Un accord de protection des investissements est déjà en vigueur depuis décembre 1992. Lors de la visite de juillet 1993 , les deux pays ont convenu d'entamer des négociations en vue d'éviter les cas de double imposition et pour protéger la propriété intellectuelle. Un accord a été conclu sur le commerce et la coopération économique qui prévoit que les deux pays s'accorderont mutuellement le traitement de la nation le plus favorisé en matière de droits de douane. La Suisse a accordé au Viêt-nam une aide financière de $\mathbf{4 0}$ millions de francs pour lui permettre de développer son secteur bancaire. Le système politique vietnamien est fondé sur le socialisme, cependant, dans le cadre de la réforme économique, les prix et les cours de change ont été libéralisés et la propriété privée est désormais autorisée. La Suisse espère pouvoir exporter et investir dans le secteur de la chimie et de la pharmacologie. Les Etats-Unis ont assoupli leur embargo économique à l'encontre du Viêt-nam, mais, même s'il est moins strict, il a encore été prolongé d'une année à partir de septembre 1993.

\section{Pays de l'Est}

En avril 1993, une délégation emmenée par le Conseiller fédéral Otto Stich s'est rendue dans les Républiques d'Asie centrale (Etats de la CEI) que sont le Turkménistan, l'Ouzbékistan, la Kirghizie et l'Azerbaïdjan. Cette visite avait pour but de mieux connaître ces pays que la Suisse représente au FMI et à la Banque mondiale. Les entretiens ont porté sur la situation politique et économique de ces pays où la réforme est en cours, sur l'établissement de relations économiques et sur les modalités d'une éventuelle coopération au développement. L'Ouzbékistan et la Suisse ont signé deux accords: un accord bilatéral économique et commercial et un accord sur la protection des investissements. En Azerbaïdjan, on a envisagé la création d'une chambre de commerce helvético-azerbaïdjanaise. Pour l'instant, la Suisse n'accorde aucune aide à ce pays. L'Azerbaïdjan et l'Arménie s'affrontent en effet dans une guerre qui a pour enjeu l'enclave arménienne du Nagorny-Karabakh et $M$. Stich a déclaré que sans ce conflit le pays n'aurait guère besoin de soutien 
financier puisqu'il possède suffisamment de ressources. Le gouvernement suisse a offert à tous ces pays d'inviter des spécialistes et des cadres pour leur permettre de suivre en Suisse des stages sur l'organisation d'une banque centrale, d'une administration financière et d'un système fiscal.

En septembre 1992, une délégation économique suisse avec l'ambassadeur Silvio Arioli a sa tête s'est rendue en Albanie, pour expliquer comment la Suisse conçoit de futures relations économiques et une coopération au développement. L'Albanie reçoit des fonds prélevés sur le crédit destiné aux pays de l'Est. Un accord de protection des investissements a été conclu dans le but de protéger les futurs investissements suisses.

\section{Amérique centrale et latine}

En octobre 1992, une délégation économique suisse s'est rendue en Argentine et au Chili. Elle avait pour objectif d'élargir le cadre des échanges commerciaux, des investissements suisses et de la coopération technique. En Argentine, les entrevues ont surtout eu pour objet le développement des relations économiques, ainsi que le problème de l'endettement extérieur. En février 1993, le président argentin Carlos Menem s'est rendu à Berne où a été signé le cinquième accord bilatéral de rééchelonnement de la dette (261 millions de francs). L'Argentine se classe deuxième, après le Brésil, parmi les pays latino-américains par l'importance des investissements suisses et des échanges commerciaux. Un accord de protection des investissements est en vigueur depuis 1992.

Au Chili, la délégation a pris part à la foire commerciale internationale tenue à Santiago. Les entretiens économiques ont porté sur la collaboration future des deux pays en matière de protection de l'environnement selon la notion d'un développement durable. Les entreprises suisses ayant consenti d'importants investissements au Chili, un accord de protection des investissements a été signé en 1991, mais il n'est pas encore entré en vigueur.

La Bolivie est l'un des principaux pays bénéficiaires de la coopération suisse au développement; les relations commerciales sont encore peut développées, mais devraient être élargies à l'avenir. Pour favoriser ce développement, les deux pays ont fondé en avril 1993 la chambre de commerce helvético-bolivienne à l'occasion d'une visite de travail en Suisse du président bolivien Jaime Paz Zamoral. Lors de cette visite, un accord de désendettement a été signé, selon lequel la Confédération renonce à des créance d'un total de 53 millions de francs (voir chapitre 1.7). Des négociations ont également été entamées en vue de conclure une convention de double imposition. Un accord de protection des investissements est en vigueur depuis mai 1991.

En été 1993 (du 1er juillet au 27 août), le Conseil de sécurité de l'ONU a décrété des sanctions économiques temporaires contre Haïti. Elles comprenaient un embargo sur les armes et un embargo sur le pétrole, ainsi que le gel des avoirs haïtiens. Ces sanctions visaientà mettre le pays sous pression pour l'inciter à organiser rapidement des élections démocratiques. La Suisse s'est associée à cet embargo. 


\section{Afrique}

La Suisse n'entretient pas une diplomatie commerciale active avec les pays d'Afrique noire. La visite d'une importante délégation au Ghana en 1991 constituait plutôt une exception (cf. Annuaire 1993). Les visites mutuelles servent essentiellement à régler des questions de coopération au développement. Le président de la République du Cap-Vert a séjourné à Berne en mai 1993 et le premier Ministre du Rwanda s'y est rendu en juin de la même année. Les entretiens ont porté sur les réformes politiques en cours dans les deux pays et sur la coopération au développement.

\section{Sources}

Office fédérale des affaires économiques extérieures Rapport sur la politique économique extérieure 1992 NZZ, 13.7.1993 (Suisse-Viêt-nam) NZZ, 26.3.1993 et Tages-Anzeiger, 17 et 19.4.1993 (Asie centrale)

NZZ, 25.9.1992 (Suisse-Albanie)

NZZ, 29.10.1992 et 2.2.1993 (Argentine, Chili)

DFAE, Communiqué de presse du 26.1.1993 (Visite de Menem à Berne)

DFAE, Communiqué de presse du 30.3.1993 (Visite du président bolivien à Berne)

NZZ, $3 / 4$ et 7.4.1993 (Suisse-Bolivie)

DFAE, Communiqué de presse du 30.6.1993 (Embargo contre Haïti)

DFAE, Communiqué de presse du 11.6.1993 (Rwanda-Suisse)

DFAE, Communiqué de presse du 18.5.1993 (Cap-Vert-Suisse)

\section{Accords de protection des investissements et conventions de double imposition}

Pour encourager les investissements étrangers, nombre de pays en développement sont prêts à offrir aux investisseurs une protection fondée en droit international. De leur côté, les pays industrialisés sont intéressés par la conclusion de tels accords, car ceux-ci améliorent la sécurité juridique des investissements et créent un climat propice au placement de capitaux. Les accords de protection des investissements régissent les transferts de capitaux et de bénéfices, le dédommagement en cas d'expropriation, ainsi que le règlement de différends. Les conventions de double imposition règlent la taxation du capital, des revenus et de la fortune et servent à éviter qu'ils ne soient imposés dans deux pays. Ces deux types d'accords du droit international sont réciproques, c'est-à-dire qu'ils s'appliquent dans les deux pays contractants.

La majeure partie des investissements directs de la Suisse dans les pays en développement se concentre sur quelques pays d'Amérique latine qui sont économiquement forts, soit le Brésil, le Mexique et l'Argentine. Après 1981, les investissements suisses dans les pays en développement ont fortement diminué pour ne s'accroître qu'à partir de 1988. Ils ont atteint 2,2 milliards de francs en 1988 
et 1989, 4 milliards en 1990 et 2,1 milliards en 1991. La répartition régionale peut se résumer comme suit: $72 \%$ en Amérique centrale et du Sud, $18 \%$ en Asie, $7 \%$ en Europe et $3 \%$ en Afrique (1). C'est surtout avec des pays en développement que la Suisse a signé des accords pour stimuler les investissements et leur assurer une protection réciproque, tandis que les conventions de double imposition sont souvent conclues avec des pays industrialisés.

\section{Protection des investissements}

Les accords de protection des investissements ont pour but de contribuer à créer des conditions stables pour les investissements dans les pays contractants. La Suisse a signé ses premiers accords de protection des investissements au début des années 60 avec des pays d'Afrique. Outre la protection des investissements, ces premiers accords contenaient aussi des dispositions sur le commerce et sur la coopération au développement (coopération technique). Depuis lors, la Suisse s'est dotée d'un réseau, relativement dense par rapport à d'autres pays, d'accords de protection des investissements: 59 accords ont été signés avec des pays en développement et des pays de l'Est, dont 53 sont en vigueur (état au 1.9.1993). Du point de vue régional, les accords de protection des investissements se répartissent comme suit: 26 avec des pays africains, 11 avec des pays d'Amérique centrale et du Sud et dix avec des pays d'Asie; 11 avec des pays de l'Est et 1 avec un pays européen (Malte). La volonté de conclure des accords de protection des investissements s'accroît également du côté des pays en développement, puisque la libéralisation de la législation sur les investissements fait en général partie des exigences du FMI et de la Banque mondiale dans le cadre de leurs programmes d'ajustement structurel.

En 1992 sont entrés en vigueur des accords de protection des investissements passés avec les pays suivants: Cap-Vert, Argentine, Paraguay et Viêt-nam; en 1993, ce fut le cas d'accords passés avec l'Albanie, l'Estonie, la Lettonie et la Lituanie (état au 1.9.1993). Aucun accord de protection des investissements n'a encore pu être signé avec le Brésil et le Mexique, qui sont les principaux partenaires économiques de la Suisse en matière d'investissements directs. L'accord signé avec l'Argentine a été précédé de négociations laborieuses.

Pays de l'Est: Un accord de protection des investissements passé avec l'ex-Union soviétique est entré en vigueur juste avant la dissolution de cette dernière en août 1991. Après cette dissolution, il restait à déterminer le champ d'application de ce contrat international. Quelques Etats nés de l'ex-URSS ont fait part de leur désir de conclure des contrats séparés; ce fut le cas des trois pays baltes - la Lituanie, la Lettonie et l'Estonie avec lesquels des accords bilatéraux de protection des investissements ont pu être signés. Ils sont entrés en vigueur en 1993. Des accords de ce type ont en outre été signés avec l'Ouzbékistan et la Biélorussie; des négociations sont en cours avec le Kazakhstan.

La négociation des accords de protection des investissements incombe à l'Office fédéral des affaires économiques extérieures, tandis que la compétence de les conclure a été déléguée en 1963 au Conseil fédéral par le Parlement. Cette délégation de compétence a ensuite été renouvelée tous les dix ans. L'arrêté fédéral actuellement en vigueur expire en février 1994; son renouvellementesten préparation. 


\section{Double imposition}

Les conventions de double imposition assurent une protection fiscale aux entreprises et aux personnes étrangères. Elles évitent que des impôts soient prélevés deux fois, c'est-à-dire dans deux pays, sur les bénéfices, les dividendes, les intérêts, les revenus et sur la fortune. Ces conventions devraient également instituer ou favoriser un climat propice aux investissements. La conclusion de telles conventions sert les intérêts de tous les pays, de sorte qu'elles ne se limitent pas, comme les accords de protection des investissements, aux relations entre pays industrialisés et pays en développement.

A la fin de 1992, 35 conventions de double imposition passées avec des pays en développement étaient en vigueur. La convention que la Suisse a conclu en 1988 avec l'ex-Union soviétique s'applique provisoirement aux Etats de la CEI, jusqu'à la négociation de conventions bilatérales. En 1993 (état au 1.9.1993), des négociations étaient en cours avec les pays en développement suivants: Inde, Pakistan (pour remplacer la convention de 1959/1962), Thaïlande, Viêt-nam, Tunisie, Turquie, Venezuela, Argentine. Des conventions ont été signées avec la Maroc et avec le Mexique.

Des discussions en vue de protéger les intérêts économiques suisses au Maroc ont déjà été menées au début des années 80 , mais elles ont été interrompues en raison de divergences de vues entre les deux pays sur le contenu de dispositions importantes de la convention et n'ont été reprises qu'en 1991. Les deux pays sont cette fois parvenus à un accord et ont signé la convention en mars 1993. Comme la plupart des conventions, celle signée avec le Maroc suit le modèle de convention de I'OCDE de 1977 et reprend les mêmes points que les conventions de double imposition passées avec d'autres pays en développement. Dans son message, le Conseil fédéral déclare que l'importance des investissements suisses au Maroc justifie que les autorités suisses concluent une telle convention avec ce pays (2). Un accord de protection des investissements est en vigueur depuis 1991.

L'élaboration des conventions de double imposition relève de la compétence de l'Administration fédérale des contributions. Une fois que la convention a été signée, l'Administration rédige un message à l'intention du Parlement qui doit la ratifier.

\section{Notes}

1. Annuaire 1993, Flux financier dans la partie statistique.

2. "L'existence d'investissements suisses relativement importants au Maroc, l'évolution récente de la politique économique de ce pays, sa proximité de l'Europe occidentale et les liens qu'il entretient avec de nombreux pays européens sont autant de raisons qui justifient, aux yeux du Conseil fédéral, la nécessité de disposer d'une convention de double imposition avec le Maroc.", Message concernant une convention de double imposition avec le Maroc, (p. 2) 


\section{Sources}

OFAEE, Liste des accords de protection de double imposition, état au 1.9.1993

Administration fédérale des contributions, Conventions suisses de double imposition, état au 1.1.1993

Rapport sur la politique économique extérieure 1992

Message sur la convention de double imposition avec le Maroc, du 12 mai 1993

NZZ, 5/6.12.1992 (Accords avec le Kazakhstan et l'Ouzbékistan)

NZZ, 28.12.1992 (Accord de libre-échange avec les pays baltes)

\section{Exportations d'armes dans les pays en développement}

Le marché international des armes et autre matériel de guerre connaissant une tendance à la baisse, ilen va de même pour les exportations suisses dans ce secteur. En 1992, les exportations suisses ont ainsi diminué de 20\% par rapport à l'année précédente, ce quicorrespond environ au recul mondial. La Turquie a été le plus gros acheteur de matériel de guerre suisse. L'interprétation de la loi de 1973 sur le matériel de guerre par le Conseil fédéral fait l'objet de vives critiques, car la Suisse achemine du matériel de guerre vers des pays du Sud-Est asiatique et du Proche-Orient où règnent des conflits armés et où, contrairement aux autres régions du monde, l'effort d'armement est soutenu. Une révision de la loi visant une interprétation plus restrictive est en préparation. Elle a été entamée sous la pression de l'initiative pour l'interdiction d'exporter du matériel de guerre qui sera probablement soumise au peuple en 1995.

Ces dernières années, le commerce mondial d'armes et autre matériel de guerre a enregistré une tendance à la baisse. Cette tendance est en partie à mettre sur le compte du désarmement, mais les problèmes budgétaires contribuent sans doute aussi à réduire le volume des transactions. Les pays du Sud-Est asiatique et du ProcheOrient font exception, car ils soutiennent un gros effort d'armement. Les exportations mondiales d'armement ont représenté entre 1987 et 1991 une valeur totale de 174 milliards de dollars, la part de la Suisse pour cette période s'est élevée à 0,9\% (1).

\section{Exportations en 1992}

En 1992, la Suisse a exporté des armes pour un montant total de 259 millions de francs, soit $20 \%$ de moins que l'année précédente. La Turquie a été son principal client; en effet, après la levée de l'embargo sur les armes (janvier 1991 - mars 1992), elle a acheté des systèmes de défense anti-aérienne au holding Oerlikon-Bührle pour plus de 60 millions de francs. Ces systèmes ont été montés en Allemagne sur des bateaux de guerre turcs. Les autorités turques et le Conseil fédéral ont affirmé que ces bateaux ne servaient pas à opprimer la minorité kurde. Le PSS et le Comité pour le désarmement etl'arrêt des exportations d'armes (ARW) ne sont pas du même avis. Ils estiment en effet qu'en raison de la guerre civile menée contre la population 
kurde dans l'Est du pays, la Turquie devrait être classée parmi les zones de conflit et que toute exportation de matériel de guerre suisse devrait être interdite vers ce pays.

La part des pays en développement dans les achats d'armement suisse s'est montée en 1992 à $40 \%$ environ. En raison de la grosse commande de la Turquie, cette part est nettement plus élevée par rapport aux années précédentes (1991: 25\%, 1990: 16\%, 1989: 34\%).

Tenant compte de l'évolution politique au Chili, le Conseil fédéral a levé en octobre 1992 l'embargo sur les armes appliqué contre ce pays. Pendant les vingt années qu'a duré l'embargo, la SIG (Schweizerische Industriegesellschaft) a fait produire au Chili des fusils d'assaut sous licence. La loi sur le matériel de guerre ne régit pas les concessions de licence.

\section{L'interprétation de la loi sur le matériel de guerre vivement critiquée}

La loi sur le matériel de guerre de 1973 prescrit que les exportations suisses de ce matériel sont exclues vers des régions où règnent des tensions. Depuis lors, la définition de ces zones de conflit fait l'objet de vives disputes. Des pays tels que le Guatemala, le Chili, I'Afrique du sud, la Chine, l'Arabie saoudite, où Amnesty International dénonce sans cesse des violations des droits de l'homme, comptent ainsi parmi les acheteurs de matériel de guerre suisse, aussi bien que des pays, tels que la Turquie, où règne la guerre civile.

Pour mettre un terme à cette dispute sur l'interprétation de la loi et pour obliger les fabricants d'armes à se convertir à une production civile, le PSS et l'ARW ont déposé en septembre 1992 I'"Initiative populaire pour l'interdiction d'exporter du matériel de guerre". Cette initiative a pour but d'interdire l'exportation et le transit de matériel de guerre, ainsi que la médiation dans le cadre de transactions de ce type. L'interdiction devrait également frapper les sociétés de financement et les biens civils pouvant être utilisés à la fabrication de matériel de guerre (2). L'initiative sera probablement soumise au peuple en 1995. Pressé par cet initiative, le Conseil fédéral prépare une révision de la loi sur le matériel de guerre dans le but de rendre son application plus restrictive. La notion de matériel de guerre devrait notamment être définie avec plus de précision.

\section{Avions d'entraînement du type PC utilisés à des fins militaires contre la population civile}

Depuis des décennies, Oerlikon-Bührle produit dans ses ateliers Pilatus à Stans des avions d'entraînement du type Pilatus Porter (PC-6, PC-7, PC-9) pour les exporter dans le monde entier. Ces avions sont considérés comme appareils civils et ne sont pas soumis à la loi fédérale sur le matériel de guerre. Dans divers pays, ces avions ont toutefois été équipés d'armes et utilisés contre la population civile. Des Pilatus achetés par les Etats-Unis sontainsi intervenus dans laguerre au Laos. Ces révélations ont provoqué en 1969 un premier scandale sur la possibilité d'utiliser ces appareil à des fins guerrières. L'armement d'appareils Pilatus et leur intervention contre la population civile (et contre des avions de la Croix-rouge) il y a plus de vingt ans dans la guerre du Biafra a également fait beaucoup de bruit. En 1973, le Conseil fédéral a promulgué la loi sur le matériel de guerre qui est encore en vigueur aujourd'hui. Elle 
interdit toute livraison de matériel de guerre lorsqu'il "apparaît que ces livraisons à un pays donné risquent de compromettre les efforts de la Confédération dans le domaine des relations internationales, notamment en ce qui concerne le respect de la dignité humaine, l'aide humanitaire ou l'aide au développement" (3). Malgré cela, on a pu démontrer que des appareils Pilatus ont continué à être utilisés contre la population, par exemple contre les Indiens au Guatemala, dans la guerre entre I'Iran et I'Irak, contre les chi'ites et les kurdes en Irak.

Au cours de la période considérée, les ventes ci-après d'appareils PC ont fait l'objet de controverses publiques:

- En Birmanie, une dictature militaire se maintient au pouvoir en bafouant les droits civils des minorités ethniques, notamment des "Karen". Depuis des années, le régime totalitaire garde la lauréate du prix Nobel de la paix Aung San Suu Kyi en résidence surveillée et interdit l'opposition. Dans le cadre de la Commission pour les droits de l'homme de l'ONU, la Suisse a voté pour une résolution contre la Birmanie. Un boycott ayant été décrété contre la Birmanie au niveau international (CE, EtatsUnis), la DDA envisage de mettre un terme à son aide bilatérale à ce pays et les oeuvres d'entraide privées de Suisse soutiennent les minorités ethniques etl'opposition démocratique birmane. Selon les révélations parues dans la presse (4), la Birmanie a été le premier pays à avoir commandé des avions PC-7 dans les années 70 , qui étaient accompagnés d'une documentation présentant explicitement la possibilité de les armer. En 1985, la Birmanie a encore acheté d'autres appareils PC (du type PC-9). En février 1993, le Comité pour le désarmement et l'arrêt des exportations d'armes révèle que des techniciens de la maison Pilatus se sont rendus en Birmanie en 1987 pour collaborer à des essais armés d'appareils PC-7 et PC-9. La presse a également rapporté en 1993 que des villages des Karen situés au Nord de la Birmanie ont été bombardés par des appareils Pilatus. En mars 1993, le Conseil fédéral a ordonné des "modifications techniques", qui doivent empêcher que les avions d'entraînement puissent être équipés d'armes après leur achat et utilisés à des fins militaires.

- Afrique duSud: La"modification technique", que le Conseil fédéral exige désormais pour tous les avions PC, doit garantir un usage exclusivement civil. Cette "garantie" a paru suffisante au Conseil fédéral pour qu'il autorise la livraison de 60 appareils PC7 à l'armée sud-africaine. Le contrat portant sur un montant de quelque 250 millions de francs a été conclu en décembre 1992 et il a été expressément approuvé par le Conseil fédéral en juin 1993. La moitié environ des travaux de finition devraient être exécutés en Afrique du Sud. Le comité des sanctions du Conseil de sécurité des Nations Unies a lancé deux appels publics au gouvernement suisse lui demandant d'interdire la vente d'avions prévue à l'aviation sud-africaine. Le Conseil fédéral a estimé que la vente de biens civils ne viole pas l'embargo de l'ONU sur les livraisons d'armes à l'Afrique du Sud (résolution 418 de 1977) et que la résolution du Conseil de sécurité 591 de 1986, qui interdit expressément la livraison d'avions, ne constitue qu'une recommandation politique et ne tient donc pas lieu de droit contraignant pour la Suisse qui n'est pas membre de l'ONU. Voici ce qu'il a de plus écrit: "Les mesures 
prises par le Conseil fédéral l'ont toujours été de manière autonome. Le Conseil fédéral a déterminé en chaque occasion si et sous quelle forme il entendait participer aux sanctions de l'ONU." (5) Considérant en particulier la loi suisse en vigueur et le fait que la transaction porte sur des avions non armés, il a estimé que la vente ne tombe ni sous le coup de la loi fédérale sur le matériel de guerre ni sous celui de l'embargo de l'ONU. La garantie contre les risques à l'exportation couvre 45 millions de francs de cette transaction.

L'attitude des autorités suisses a provoqué un tollé aussi bien en Suisse qu'à l'étranger. Le Mouvement anti-apartheid de Suisse (MAAS) a protesté et fait observer que "... la société Pilatus est le seul producteur d'armes au monde qui fournit aujourd'hui à l'armée d'apartheid des avions d'entraînement pour ses pilotes de chasse". Selon le MAAS, Pilatus a remporté le marché après que l'Afrique du Sud a rompu des négociations avec une firme concurrente brésilienne, car celle-ci exigeait des conditions garantissant que les appareils ne puissent pas servir à des fins militaires. Le Congrès national africain (ANC) s'était prononcé contre cet achat et déclaré en octobre 1992 déjà qu'il ne se porterait pas garant des contrats, ni de leurs conséquences financières, qui aurontété conclus en désaccord avecl'embargo sur les livraisons d'armes de l'ONU (6). L'ANC a notamment recommandé à la Suisse d'attendre la tenue d'élections en Afrique du Sud et la formation d'un gouvernement de transition avant de conclure ce contrat. Le PSS, I'Union syndicale suisse, la Communauté de travail des oeuvres d'entraide et Amnesty International Suisse ont également protesté contre cette vente d'avions Pilatus à l'Afrique du Sud.

En 1993, les ateliers Pilatus ont livré sept appareils PC-6 à une entreprise privée d'Afrique du Sud qui les équipe pour la police sud-africaine. L'appareil du type PC6 est produit depuis plus de 30 ans et ses qualités civiles ne sont pas mises en doute. II n'est pas réputé être un avion de guerre potentiel.

- Corée du Sud: En juillet 1993, la presse a rapporté que la Corée du Sud insiste pour que les 20 PC-9 qu'elle a prévu d'acheter soient équipés des points d'attache prévus pour armer l'appareil. Depuis le milieu des années 50 , la Suisse considère la Corée du Sud comme une zone de conflit dans laquelle toute livraison d'armes est interdite. Le Conseil fédéral s'est déclaré prêt à autoriser la livraison à condition que soit respectée sa décision de mars 1993 excluant toute possibilité d'armer ultérieurement des avions Pilatus. En septembre 1993, le Conseil fédéral a cependant autorisé l'exportation des 20 avions PC-9 "dans la configuration originale" (points d'attache et dispositif de largage compris).

A l'initiative demandant l'interdiction d'exporter des armes, le Conseil fédéral oppose une révision de la loi sur le matériel de guerre, qui pourrait entre autres classer les avions PC-7 et PC-9 parmi le matériel de guerre. De son côté, la société Pilatus a aussi décidé d'agir et renvoyé en mars 1993 le président de sa direction (Walter Gubler), qui savait que les Pilatus PC-7 et PC-9 étaient utilisés comme avions de combat, mais n'en n'avait pas moins cessé les livraisons dans des pays peu sûrs, tels que la Birmanie, l'Irak, l'Angola, le Mexique et le Chili. L'équipement militaire des appareils était en général assuré dans le pays acheteur par des entreprises françaises et belges, qui en avisaientla directions de Pilatus (7). La nouvelle direction 
a annoncé l'adoption d'une nouvelle politique commerciale, selon laquelle la société refuserait de traiter avec les pays qui souhaitent destiner les avions Pilatus à des fins militaires. En 1993, ce fut le cas du Guatemala, du Sri Lanka et des pays nés de l'éclatement de la Yougoslavie. En août 1993, legroupe Oerlikon-Bührle, propriétaire des usines Pilatus, a publié cette nouvelle politique dans un livre blanc sur l'exportation des avions d'entraînement ("Weissbuch zur Frage der Ausfuhr von Trainingsflugzeugen") (8).

Tableau $\mathrm{N}^{\circ} 14$

\section{Exportations suisses de matériel de guerre 1991/1992 \\ Les 15 principaux pays en développement clients de la Suisse}

\begin{tabular}{|c|c|c|c|c|c|}
\hline \multirow[b]{2}{*}{ Pays' } & \multicolumn{2}{|c|}{1991} & \multirow[b]{2}{*}{ Pays' } & \multicolumn{2}{|c|}{1992} \\
\hline & $1000 \mathrm{Fr}$. & en $\%$ & & $1000 \mathrm{Fr}$. & en $\%$ \\
\hline Malaisie & $53 ' 937$ & 16,5 & Turquie & $63 ' 505$ & 24,5 \\
\hline Singapour & $13 ' 569$ & 4,1 & Singapour & $12 ' 335$ & 4,8 \\
\hline Brésil & 1'585 & 0,5 & Arabie saoudite & 8'945 & 3,5 \\
\hline Chine & 814 & 0,2 & Malaisie & 4'527 & 1,7 \\
\hline Corée du Sud & 695 & 0,2 & Pakistan & $1 ' 154$ & 0,4 \\
\hline Pakistan & 651 & 0,2 & Brésil & $1 ' 023$ & 0,4 \\
\hline Indonésie & 511 & 0,2 & Egypte & 806 & 0,3 \\
\hline Maroc & 414 & 0,1 & Maroc & 621 & 0,2 \\
\hline Emirats arabes & 399 & 0,1 & Corée du Sud & 533 & 0,2 \\
\hline Algérie & 357 & 0,1 & Emirats arabes & 491 & 0,2 \\
\hline Turquie & 314 & 0,1 & Inde & 430 & 0,2 \\
\hline Nigeria & 258 & 0,1 & Algérie & 354 & 0,1 \\
\hline Arabie saoudite & 249 & 0,1 & Croatie & 130 & 0,1 \\
\hline Colombie & 139 & 0,0 & Taïwan & 129 & 0,0 \\
\hline Thaillande & 132 & 0,0 & Colombie & 125 & 0,0 \\
\hline & & & Botswana & 107 & 0,0 \\
\hline $\begin{array}{l}\text { Autres pays en } \\
\text { développement }\end{array}$ & 735 & 0,2 & $\begin{array}{l}\text { Autres pays en } \\
\text { développement }\end{array}$ & 843 & 0,3 \\
\hline $\begin{array}{l}\text { Total des pays } \\
\text { en développement }\end{array}$ & $74^{\prime} 558$ & 22,8 & $\begin{array}{l}\text { Total des pays } \\
\text { en développement }\end{array}$ & $95 ' 215$ & 36,88 \\
\hline Pays industrialisés & $252 ' 437$ & 77,2 & Pays industrialisés & $162 ' 701$ & 62,9 \\
\hline Exportations totales & $326 ' 995$ & 100,0 & Exportations totales & $258 ' 759$ & 100,0 \\
\hline
\end{tabular}

1) Pays de destination finale

Source: Calculé à partir des chiffres fournis par l'Administration militaire fédérale. 


\section{Livraisons d'armes dans les zones de conflit}

Indonésie: En Indonésie, le président Suharto fait régner depuis plus de vingt ans un régime dictatorial qui viole systématiquement les droits de l'homme, comme l'a démontré Amnesty International (9). Dans I'Etat pluri-ethnique qu'est I'Indonésie, les minorités sont poursuivies et opprimées, en particulier sur l'île de Sumatra et au Timor Oriental. L'Indonésie a envahi ce territoire en 1975 et, depuis lors, plus de 200'000 personnes, selon Amnesty International, sont mortes pour s'être opposés à l'occupant indonésien. Ces dernières années, I'Indonésie a connu une forte croissance économique fondée sur une politique économique restrictive orientée vers l'exportation. A cela il faut ajouter les déboisements massifs pratiqués dans la forêt tropicale en Papouasie occidentale, qui privent les indigènes de leurs bases vitales. Jusqu'à présent, la Suisse n'a pas exporté d'armes vers I'Indonésie. En juillet 1993 cependant, le Conseil fédéral - en raison de son caractère explosif la décision a en effet été soumise au Conseil fédéral-a donné un préavis positif à une demande d'Oerlikon-Contraves àZurich, qui souhaite livrer en Indonésie des canons antiaériens pour une valeur de 10 millions de francs. Comme pour les livraisons destinées à la Turquie en 1992, le Conseil fédéral a motivé sa décision en arguant que les canons antiaériens sont des armes défensives et qu'ils ne seraient pas utilisés contre la population civile. On ne distingue toutefois pas le matériel de guerre selon son utilisation (armes offensives ou défensives). Les personnes qui ont critiqué cette décision craignent que l'autorisation de livrer des armes à l'Indonésie ne crée un précédent pour de futurs contrats d'armement. L'Indonésie compte parmi les pays qui se livrent à une course effrénée à l'armement en Asie en vue de s'arroger une place militaire prépondérante sur ce continent. Le PSS a notamment souligné que la décision du Conseil fédéral contrevient de toute évidence à la loi, puisqu'il a autorisé des livraisons d'armement suisse dans une zone de conflit internationalement reconnue.

\section{Interdiction d'exporter et contrôle des exportations}

L'initiative pour une interdiction d'exporter des armes a été lancée en Suisse à l'occasion de la guerre du Golfe en 1991. A cette époque, I'ONU avait dressé une liste des installations atomiques irakiennes où 134 machines sur 602 et environ un quart des équipements "critiques" provenaient de Suisse. Les exportations suisses d'armement ne représentent que $0,28 \%$ des exportations globales (1992), tandis que la Suisse est le troisième exportateur mondial de machines-outils (derrière l'Allemagne et le Japon). Répondant aux voix qui se sont élevées au niveau international pour critiquer la participation suisse à l'armement de l'Irak, le Conseil fédéral a adopté une ordonnance prévoyant que les machines-outils et tous les produits pouvant aussi servir à des fins militaires (les biens dits à double usage) seraient soumis au contrôle des exportations (10). Il a également renforcé le contrôle des exportations de biens et de technologies pouvant servir à la fabrication d'armes $\mathrm{ABC}$ (atomiques, biologiques et chimiques). La nouvelle ordonnance est en vigueur depuis mars 1993 et elle comprend aussi une nouvelle liste, sensiblement plus longue, des machines-outils soumises au contrôle des exportations. Enjanvier 1993, 
la Suisse a signé la Convention sur les armes chimiques, qui a pour but d'éliminer les armes chimiques dans le monde entier.

\section{Registre de l'armement de l'ONU}

L'Assemblée générale de l'ONU a décidé en 1991 de tenir un registre international sur le commerce d'armes conventionnelles. Les exportations des armes concernées ont pour la première fois été enregistrées en 1992. Dès 1994, on enregistrera aussi, outre les exportations et les importations d'armes, la production indigène destinée à l'armée nationale, ce qui permettra de connaître exactement (par catégories) l'équipement militaire d'un pays. Pratiquement tous les pays ont collaboré à l'établissement de ce registre, même certains pays du Proche-Orient ont fourni des statistiques sur leur commerce d'armement. La tenue de ce registre a pour objectif de créer la transparence dans ce domaine et de stimuler le désarmement.

\section{Notes}

1. NZZ du 2.7.1993 (Initiative pour une interdiction d'exporter du matériel de guerre)

2. Pour plus d'informations sur l'initiative, voir l'Annuaire 1992, p. 109 et suiv.

3. Loi fédérale sur le matériel de guerre du 30 juin 1972, art. 11, al. 2 b).

4. mosquito $n^{\circ}$ 7/septembre 1992 et Friedenspolitik no 67/mars 1993.

5. DFAE, Communiqué de presse du 3.2.1993 (Réponse de la Suisse au comité des sanctions du Conseil de sécurité des Nations Unies concernant la livraison d'avions d'entraînement Pilatus PC-7 à l'Afrique du Sud).

6. Anti-Apartheid-Nachrichten $n^{\circ}$ 6/décembre 1992: Stanser Hilfe für Apartheidgeneräle.

7. Le Nouveau Quotidien, 21.3.1993, Tages-Anzeiger et NZZ du 27.3.1993.

8. NZZ, 25.8.1993 (Présentation de la politique commerciale de Pilatus dans le "Livre blanc").

9. Amnesty International Magazin $n^{0}$ 9/septembre 1993: Indonesien - Suharto eiserne Hand.

10. Tages-Anzeiger du 5.8.1993 (Interdiction d'exporter des armes).

\section{Sources}

Comité pour le désarmement et l'arrêt des exportations d'armes (éd.), Friedenspolitik no 67/mars 1993 et no 68/juin 1993

epd-Entwicklungspolitik 14/93 (juillet), Das UNO-Waffenregister

ai-Magazin 12/92, Schweizer Waffen für Chile

Tages-Anzeiger, 3.12.1993 (Des avions Pilatus pour l'aviation sud-africaine)

Tages-Anzeiger, 2.6.1993 (Expertise juridique: la livraison des Pilatus pose problème/ garantie GRE pour le contrat avec l'Afrique du Sud)

NZZ, 4.2.1993 (Exportation d'appareils PC-7 autorisée)

NZZ, 2.6.1993 (Garantie GRE accordée pour la livraisons des PC-7)

Tages-Anzeiger, 10.7.1993 et NZZ, 13.7.1993 (Livraisons d'armes à l'Indonésie)

Tages-Anzeiger et NZZ du 16.7.1993 (Des PC-9 pour la Corée du Sud)

NZZ, 18.2.1993 (Extension des contrôles d'exportations d'armes)

NZZ, 27/28.3.1993 et Tages-Anzeiger, 27.3.1993 (La moralité des ateliers Pilatus)

Tages-Anzeiger, 31.7.1993 (Des PC-6 pour la police sud-africaine)

NZZ, 15.1.1993 (Signature de la Convention sur les armes chimiques) 